\section{Mit Prothesen den Phantomschmerz besiegen}

\author{
Menschen mit (Teil-) Verlust einer Extremität leiden in den meisten \\ Fällen unter Phantomschmerzen. Neuroforscher haben die amputa- \\ tionsbedingten kortikalen Umbauprozesse teilweise entschlüsselt und \\ daraus viel versprechende Therapieansätze entwickelt, die auf einer \\ Kombination von High-Tech und sensomotorischen Übungen basieren.
}

$P$ rofessor Thomas Weiß et al. haben am Institut für Psychologie der Universität Jena eine myoelektrische Prothese mit sensorischem Feedback entwickelt. Sensoren in der künstlichen Hand melden die Griffstärke an Stimulationselektroden, die auf der Oberarmhaut des Patienten platziert sind. Wer fleißig übt, bekommt damit auch fragile Gegenstände unbeschadet in den Griff. Das eigentliche Interesse der Forscher gilt aber der Frage, ob damit Phantomschmerzen reduziert werden können. Im Prinzip ja: Nach einem zweiwöchigen Training steigerten acht Patienten nicht nur ihre Geschicklichkeit mit der Prothese, sondern erfuhren auch eine Linderung ihrer Phantomschmerzen um durchschnittlich $48 \%$ [1].

Phantomschmerzen treten oft in engem Zusammenhang mit kortikalen Reorganisationsprozessen auf. Dabei werden die dem amputierten Glied zugeordneten somatosensorischen Kortexareale von benachbarten Arealen mitbenutzt. Infolge solcher zentralen Reorganisationsprozesse kann es auch zu Fehlwahrnehmungen kommen. Dazu zählen unter anderem übertragene Empfindungen, etwa die verkürzte Wahrnehmung des Phantomglieds - das Teleskopphänomen.

Ausgangspunkt der Studie war die These, dass der Prozess der Reorganisation durch gezieltes Üben wieder umgekehrt werden könnte. Man versucht dabei gewissermaßen, den kortikalen Platz, der durch die Amputation frei geworden ist, wieder regulär zu besetzen - durch die Prothese, die es möglichst gut ins Körperschema des Patienten zu integrieren gilt. Für die Integration eines Glieds und die daran gekoppelte Wahrnehmung „das ist meine Hand" scheint eine bilaterale Aktivierung von parietalen und prämotorischen Kortexarealen essenziell zu sein, so das Ergebnis einer fMRT-Studie mit Probanden, die eine Gummihand integrieren sollten [2] (Abbildung 1).

\section{Teleskop als virtuelle Realität}

Bereits frühere Studien hatten gezeigt, dass sowohl mit dem Verwenden einer Prothese als auch mit sensorischen Feedbackverfahren eine ausgeprägte Reduktion von Phantomschmerzen bewirkt werden kann. Auch imaginierte Bewegungen des Phantomglieds führen bereits zu Schmerzlinderung und einer „ReReorganisation“ der Hirnaktivierung. fekt bei der Spiegeltherapie zu sein. Professor Herta Flor, Institut für Neuropsychologie und Klinische Psychologie, Universität Heidelberg-Mannheim, vermutet, dass dabei die Kombination von motorischem und sensorischem Training besonders günstig auf die kortikale Integration des Phantoms wirken.

Bei Amputierten mit Teleskop sind die Phantomschmerzen umso stärker, je kürzer das wahrgenommene Phantom ist. Das erschwert es dem Patienten, etwa im Rahmen der Spiegeltherapie oder auch beim Tragen einer Prothese, das Gesehene mit dem gefühlten Gliedmaß in Kongruenz zu bringen. Dieses Problem könne man nun mithilfe der virtuellen Realität umgehen, berichtet Flor. Damit kann man nun jede erdenkliche Form von Teleskop simulieren. Thomas Bißwanger-Heim

\section{Literatur:}

1. Dietrich C et al. Neurosci Lett 2012; 507: 97-100

2. Ehrsson HH et al. Science 2004; 305: 875-7

Symposium „Beeinflusst die intakte Körperwahrnehmung die Behandlung von Phantomschmerz? Erweiterte Realität und Prothesentraining als innovative Behandlungsmethoden", 20.10.2012, Deutscher Schmerzkongress 2012, Mannheim Besonders ausgeprägt scheint dieser Ef-
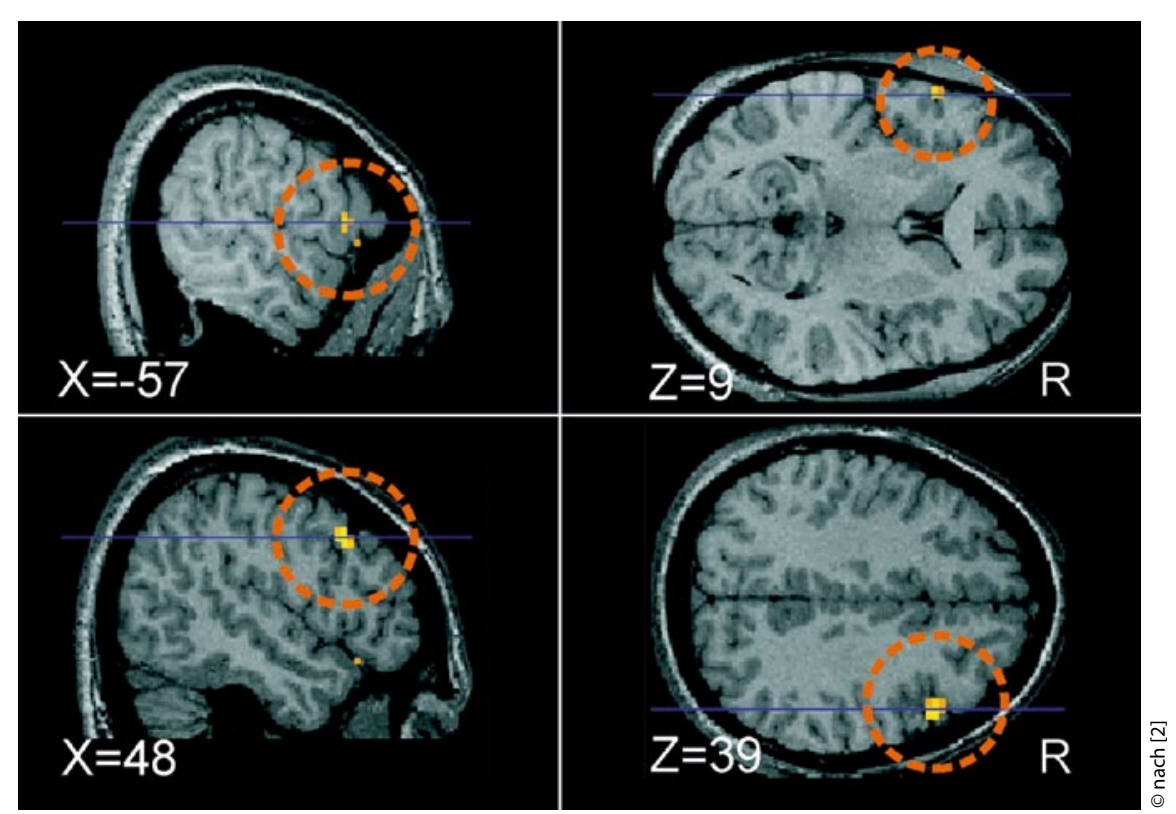

Abbildung 1: „Das ist meine Hand“: Diese Integrationsleistung geht mit einer bilateralen Aktivierung von prämotorischen und parietalen Kortexarealen einher. 